\title{
The Emotional and Professional Costs of Pleasing Customers
}

\author{
Hana Medler-Liraz and Dana Yagil
}

\begin{abstract}
The goal of this study was to explore the emotional and the professional costs incurred by service providers with a strong need to belong (i.e., craving for social acceptance, Baumeister and Leary 1995; Lavigne et al. 2011). The research model predicts that service providers with a strong need to belong will develop emotional labor strategies to please their customers, which will subsequently lead to emotional exhaustion, falling to provide high quality service, and low customer loyalty intentions.

The sample consisted of 170 restaurant servers and customer dyads. Both parties were asked to relate to their latest service interaction. The results suggest that the service providers' need to belong is positively related to emotional labor strategies, which are positively related to emotional exhaustion. Emotional exhaustion is negatively related to customers' loyalty intentions. Furthermore, emotional labor strategies mediate the relationship of the need to belong with emotional exhaustion.

The study sheds light on the complexity of service providers' motivation to please customers. On the one hand, recruiting service providers who are internally motivated to please the customers appear to be desirable in the service context. On the other hand, such service providers are at a higher risk of burnout which might ultimately undermine business profitability.
\end{abstract}

References available upon request.

H. Medler-Liraz ( $\triangle)$

Academic College of Tel-Aviv-Yaffo, Tel Aviv, Israel

e-mail: hanamedl@mta.ac.il

D. Yagil

University of Haifa, Haifa, Israel

e-mail: dyagil@ research.haifa.ac.il 